\title{
A systemic review and meta-analysis of the clinical efficacy and safety of total glucosides of peony combined with methotrexate in rheumatoid arthritis
}

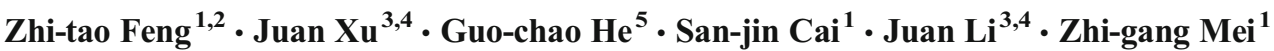

Received: 14 March 2017 / Revised: 8 July 2017 / Accepted: 18 July 2017 /Published online: 27 July 2017

(C) The Author(s) 2017. This article is an open access publication

\begin{abstract}
To assess the efficacy and safety of the combination of total glucoside of peony (TGP) and methotrexate (MTX) for the treatment of rheumatoid arthritis (RA). Randomized controlled trial (RCT) data on the traditional Chinese active component TGP combined with MTX vs. MTX alone for the treatment of RA was collected by searching the Pubmed, Embase, Cochrane Library, CNKI, VIP Journals database, and Wanfang database up to February 2017. Study selection, data extraction, data synthesis, and data analyses were performed according to the Cochrane standards. A total of eight RCTs involving 522 participants were included in this metaanalysis. Compared with MTX alone, the use of TGP combined with MTX exhibited better therapeutic effects for the treatment of RA $(P=0.004)$. In addition, TGP combined with MTX caused a more significant decrease in erythrocyte sedimentation rate $(\mathrm{ESR})(P<0.0001)$ and swollen joint count
\end{abstract}

Juan Li

1j40038@126.com

Zhi-gang Mei

meizhigang@ctgu.edu.cn

1 Third-Grade Pharmacological Laboratory on Chinese Medicine Approved by State Administration of Traditional Chinese Medicine, Medical College of China Three Gorges University, Yichang, Hubei 443002, China

2 Shenzhen Institute of Geriatrics, Shenzhen, Guangdong 518020, China

3 Department of Rheumatology, Nanfang Hospital, Southern Medical University, Guangzhou, Guangdong 510515, China

4 Department of Traditional Chinese Internal Medicine, School of Traditional Chinese Medicine, Southern Medical University, Guangzhou, Guangdong 510515, China

5 Department of Orthopedic Surgery, Hunan Provincial Hospital of Traditional Chinese Medicine, Zhuzhou, Hunan 412008, China
(SJC) $(P<0.00001)$. However, no significant differences were found in C-reactive protein (CRP) $(P=0.19)$, duration of morning stiffness (DMS) $(P=0.32)$, or tender joint count (TJC) $(P=0.23)$ between the two groups. In addition, adverse events were more frequently reported in the MTX monotherapy group than in the TGP and MTX combination group $(P=0.0007)$. Our study demonstrates that TGP combined with MTX is more effective than MTX alone for the treatment of RA. Nevertheless, the adverse effects of the combination of TGP and MTX need to be further assessed. Due to the poor methodological quality of included trials, well-designed, multi-center, and large-scale RCTs are necessary to draw a more definitive conclusion.

Keywords Meta-analysis $\cdot$ Methotrexate $\cdot$ Randomized controlled trial $\cdot$ Rheumatoid arthritis · Systematic review · Total glucosides of peony $\cdot$ Traditional Chinese medicine

\section{Introduction}

Rheumatoid arthritis (RA) is one of the most common autoimmune diseases in the world and is characterized by synovial inflammation, hyperplasia, autoantibody production, and cartilage and bone destruction [1,2]. RA can cause a loss of joint function, reduce quality of life, and enhance mortality. Approximately $1 \%$ of the worldwide population is affected by RA [3]. Its pathogenesis is not fully understood, rendering efforts towards preventative treatment ineffective [4]. The current treatment strategies for RA include nonsteroidal antiinflammatory drugs (NSAIDs), corticosteroids, diseasemodifying anti-rheumatic drugs (DMARDs), biologic response modifiers (biologicals), and traditional Chinese medicine (TCM) [5-8]. The recommended treatment model begins with DMARDs after RA diagnosis [5]. The treatment 
generally begins with methotrexate (MTX), which acts as an "anchor drug" [9]. TCM and TCM preparations have unique advantages for the treatment of RA, such as overall adjustment, multi-level, multiple targets, and fewer side effects $[10,11]$. In recent years, the potential and positive effects of TCM have increasingly attracted public interest for the treatment of autoimmune diseases.

Total glucoside of peony (TGP) is a biologically active compound extracted from roots of Paeonia lactiflora Pall [12] and is a traditional Chinese medicine that has been used to treat RA for centuries. Pharmacological studies have indicated that TGP has anti-inflammatory [13-16], immuneregulatory $[17,18]$, and analgesic effects $[19,20]$. In addition, TGP was shown to reduce joint pain and swelling and inhibit joint damage [21, 22]. Base on these potential properties, TGP (trade name: pafulin) is widely used for the treatment of RA in China. Currently, several clinical studies have demonstrated a significant improvement of RA symptoms and the prevention of RA progression afforded by TGP combined with MTX compared to MTX monotherapy. However, the results of these studies are inconsistent, and very little is known about the side effects of the combined therapy. Therefore, we conducted this meta-analysis of randomized controlled trials (RCT), in order to systematically evaluate the efficacy and safety of TGP combined with MTX vs. MTX monotherapy for the treatment of RA. We hypothesized that the results of this study could provide evidence for the superiority of treating RA with TGP plus MTX.

\section{Materials and methods}

\section{Search strategy}

The Pubmed, Embase, Cochrane Library, China National Knowledge Infrastructure (CNKI), VIP Journals database, and Wanfang databases were searched to collect published studies for the present meta-analysis. All of these databases were searched for relevant data from their inception to the latest issue (February 2017). A number of words including "rheumatoid arthritis," "RA," "total glucoside of peony," "Pafulin," "TGP," "methotrexate," and "MTX" were utilized as both medical subject heading $(\mathrm{MeSH})$ terms and text words to identify all articles having reported on the combination of TGP and MTX for the treatment of RA. A manual search of references from original research or review articles was performed to identify additional studies. No language or time restrictions were applied in the search.

\section{Study selection}

Trials were considered to be eligible for inclusion if they met all of the following criteria: (i) all patients fulfilled the
1987 revised American College of Rheumatology criteria for the disease [23], (ii) the RCTs, the evaluated efficacy, and safety of TGP combined with MTX for RA patients, (iii) outcomes included at least one of the following: therapeutic effects (TEs), erythrocyte sedimentation rate (ESR), rheumatoid factor (RF), C-reactive protein (CRP), duration of morning stiffness (DMS), swollen joint count (SJC), tender joint count (TJC), and adverse events (AE). There were no restrictions regarding gender, age, severity or duration of RA.

\section{Data extraction}

The relevant data were extracted by two independent reviewers (Zhitao Feng and Juan Xu), including study design, randomization, diagnostic criteria, the first author's name, publication year, sample size, treatment duration, dose, outcomes, and AEs. Disagreements were resolved by consensus or were arbitrated by a third investigator (Juan Li or Zhigang Mei).

\section{Statistical analysis}

Review Manager 5.3 software (Cochrane Collaboration, Oxford, UK) was used to analyze the data. The quality of each included study was assessed according to the Cochrane Handbook for Systematic Reviews of Interventions and Jadad scoring [24]. Odds ratios (OR) with $95 \%$ confidence intervals (CIs) were calculated for dichotomous data. If continuous data were available, the mean difference (MD) with $95 \%$ CIs was calculated. A fixed-effect model was employed if there was no statistical heterogeneity among studies; otherwise, the randomeffect model was used [25]. Cochrane's chi-square test was used to assess heterogeneity, and Higgins $I^{2}$ measures the degree of inconsistency between studies whether the percentage total variation across studies is due to heterogeneity rather than chance $[8,26]$. Publication bias was detected by the use of funnel plots and the Egger regression asymmetry test (Stata software, version 12.0).

\section{Results}

\section{Study selection and characteristics}

Employing the abovementioned search strategy, we retrieved 189 potentially relevant articles. After removal of duplicates, 120 studies were screened. The content review limited the relevant papers to eight studies that met the inclusion criteria for the meta-analysis [27-34]. The general procedure for study selection is shown in Fig. 1.

A total of eight trials including 265 RA cases and 257 controls that met our inclusion criteria were included in the 
Fig. 1 Flow diagram of study selection process

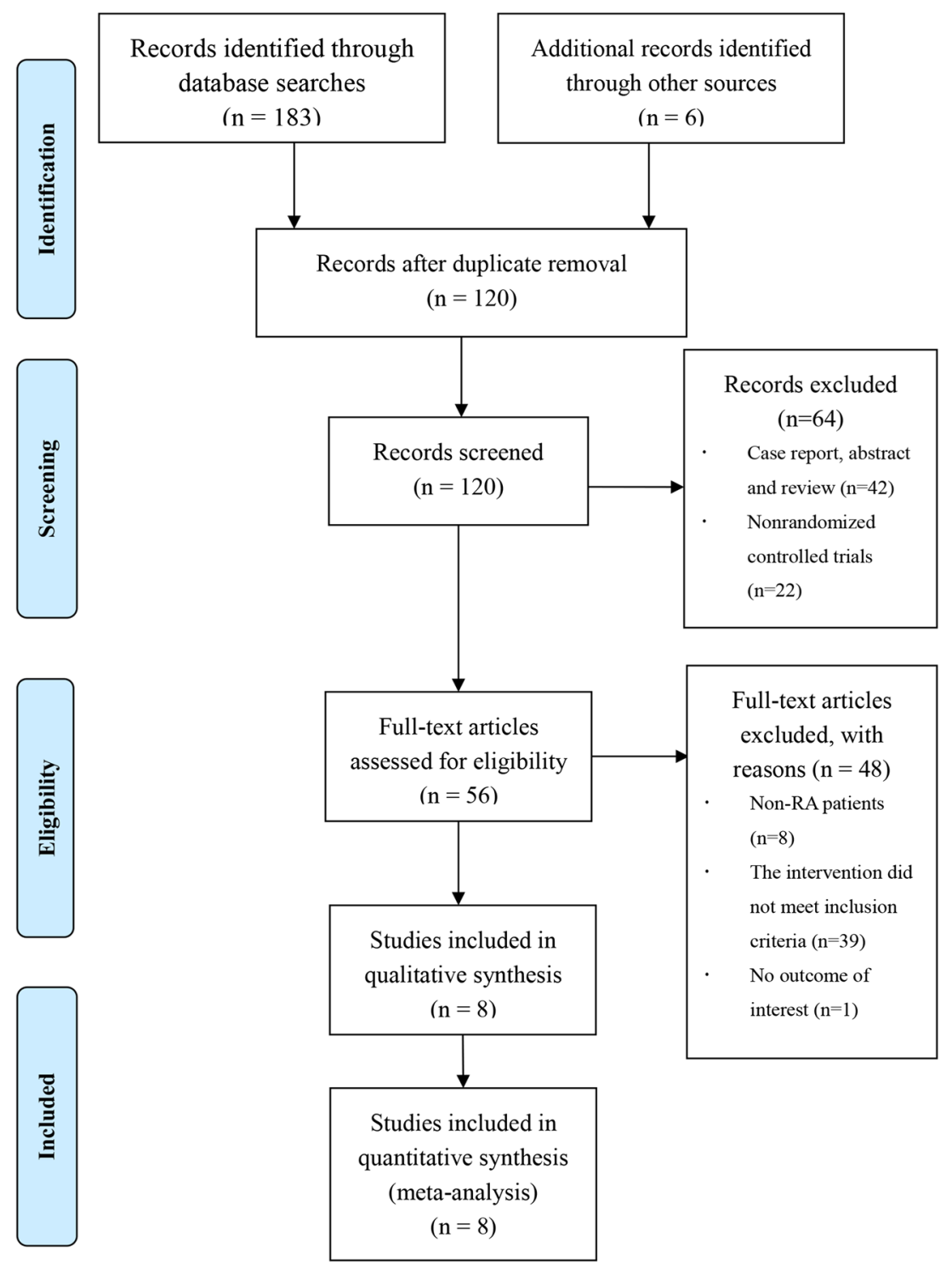

present study. The participant numbers in the individual studies varied from 46 to 90 . The TGP doses ranged between 0.9 and $1.8 \mathrm{~g} /$ day but most were $1.8 \mathrm{~g} /$ day. The dose of MTX in the combination therapy and monotherapy groups ranged between 7.5 and $15 \mathrm{mg} /$ week. The duration of the interventions in the included studies varied from 12 to 24 weeks and 6 studies described TEs evaluated on the basis of four classes of outcomes such as "cure," "significant effective," "effective," and "ineffective" [27-29, 31, 32, 34]. Four of the trials discussed the AEs in detail $[27,29,30,34]$. In addition, five mentioned the ESR [27-29, 33, 34]; two mentioned the CRP [27, 33]; one mentioned the RF [33]; four mentioned the DMS [27-29, 34]; three mentioned the TJC [28, 29, 34]; and three analyzed SJC [28, 29, 34]. The characteristics of the included RCTs are summarized in Table 1.

\section{Risk of bias assessment}

Most included RCTs exhibited poor methodological quality according to the criteria shown in Fig. 2. All the trials described the randomization procedure, but none reported allocation concealment. Insufficient information was available for assessment of the allocation method. In addition, no study described the blinding of participants or dropout information.

\section{The TEs of TGP combined with MTX vs. MTX alone}

The TEs of TGP combined with MTX vs. MTX alone were compared in six RCTs including of a total of 416 patients. As shown in Fig. 3, the pooled results from these trials showed a significant difference in the TEs between TGP combined with MTX and those of MTX alone $(P=0.004 ; \mathrm{OR}=3.70 ; 95 \% \mathrm{CI}$ 
Table 1 Characteristics of the eight trials included in the meta-analysis

\begin{tabular}{|c|c|c|c|c|c|c|c|c|}
\hline \multirow[t]{2}{*}{ Author } & \multicolumn{2}{|l|}{ Participants } & \multicolumn{2}{|l|}{ Age } & \multicolumn{2}{|l|}{ Interventions } & \multirow[t]{2}{*}{ Duration } & \multirow[t]{2}{*}{ Outcomes } \\
\hline & $\mathrm{E}(\mathrm{M} / \mathrm{F})$ & $\mathrm{C}(\mathrm{M} / \mathrm{F})$ & $\mathrm{E}$ & $\mathrm{C}$ & $\mathrm{E}$ & $\mathrm{C}$ & & \\
\hline Zhang 2010 [27] & $42(13 / 29)$ & $42(12 / 30)$ & $41.3 \pm 13.4$ & $40.9 \pm 12.3$ & $\begin{array}{l}\text { TGP } 0.3 \mathrm{~g} \text { tid, MTX } \\
10 \mathrm{mg} \mathrm{qw}\end{array}$ & $\begin{array}{l}\text { MTX } 10 \\
\text { mg qw }\end{array}$ & 24 weeks & $\begin{array}{l}\text { TE, ESR, CRP, DMS, } \\
\text { AE }\end{array}$ \\
\hline Ma 2010 [28] & 30 & 30 & NA & NA & $\begin{array}{l}\text { TGP } 0.6 \mathrm{~g} \text { bid, MTX } \\
7.5 \sim 15 \mathrm{mg} \mathrm{qw}\end{array}$ & $\begin{array}{l}\text { MTX 7.5 15 } \\
\text { mg qw }\end{array}$ & 24 weeks & $\begin{array}{l}\text { TE, ESR, DMS, } \\
\text { TJC, SJC }\end{array}$ \\
\hline $\begin{array}{l}\text { Shang et al. } 2009 \\
\text { [29] }\end{array}$ & $31(11 / 20)$ & $28(9 / 19)$ & $40 \pm 6$ & $39 \pm 6$ & $\begin{array}{l}\text { TGP } 0.6 \mathrm{~g} \text { tid, MTX } \\
7.5 \mathrm{mg} \mathrm{qw}\end{array}$ & $\begin{array}{r}\text { MTX } 7.5 \\
\text { mg qw }\end{array}$ & 12 weeks & $\begin{array}{r}\text { TE, ESR, DMS, } \\
\text { TJC, SJC, AE }\end{array}$ \\
\hline Zhu 2009 [30] & $23(6 / 17)$ & $23(7 / 16))$ & $46 \pm 12$ & $47 \pm 11$ & $\begin{array}{l}\text { TGP } 0.6 \mathrm{~g} \text { tid, MTX } \\
7.5 \mathrm{mg} \mathrm{qw}\end{array}$ & $\begin{array}{l}\text { MTX } 15 \\
\text { mg qw }\end{array}$ & 24 weeks & TE, AE \\
\hline Liu et al.2007 [31] & 46 & 44 & NA & NA & $\begin{array}{l}\text { TGP } 0.6 \mathrm{~g} \text { tid, MTX } \\
7.5 \sim 10 \mathrm{mg} \mathrm{qw}\end{array}$ & $\begin{array}{l}\text { MTX 7.5 } \\
10 \mathrm{mg} \mathrm{qw}\end{array}$ & 24 weeks & TE, AE \\
\hline $\begin{array}{l}\text { Wang et al.2007 } \\
\text { [32] }\end{array}$ & 32 & 30 & NA & NA & $\begin{array}{l}\text { TGP } 0.6 \mathrm{~g} \text { tid, MTX } \\
7.5 \mathrm{mg} \mathrm{qw}\end{array}$ & $\begin{array}{c}\text { MTX } 7.5 \\
\text { mg qw }\end{array}$ & 12 weeks & TE, AE \\
\hline Yin et al.2007 [33] & 30 & 30 & NA & NA & $\begin{array}{l}\text { TGP } 0.6 \mathrm{~g} \text { bid, MTX } \\
10 \mathrm{mg} \mathrm{qw}\end{array}$ & $\begin{array}{l}\text { MTX } 10 \\
\text { mg qw }\end{array}$ & 12 weeks & ESR, CRP, RF, AE \\
\hline Du et al.2005 [34] & 31 & 30 & NA & NA & $\begin{array}{l}\text { TGP } 0.6 \mathrm{~g} \text { tid, MTX } \\
15 \mathrm{mg} \mathrm{qw}\end{array}$ & $\begin{array}{l}\text { MTX } 15 \\
\text { mg qw }\end{array}$ & 12 weeks & $\begin{array}{l}\text { TE, ESR, DMS, } \\
\text { TJC, SJC, AE }\end{array}$ \\
\hline
\end{tabular}

$T G P$ total glucosides of peony, $M T X$ methotrexate, $T E$ therapeutic effect, $E S R$ erythrocyte sedimentation rate, $C R P C$-reactive protein, $R F$ rheumatoid factor, $D M S$ duration of morning stiffness, $T J C$ tender joint count, $S J C$ swollen joint count, $A E$ adverse event, $E$ experiment group, $C$ control group, $M$ male, $F$ female, $N A$ not available

1.51 to 9.04$)$ based on the random-effect model $\left(I^{2}=53 \%\right.$, $P=0.06)$.

\section{ESR $(\mathrm{mm} / \mathrm{h}), \mathrm{CRP}(\mathrm{mg} / \mathrm{L}), \mathrm{DMS}, \mathrm{TJC}$, and SJC}

Five trials reported the effects of the combination of TGP and MTX vs. MTX alone on ESR serum levels, and a fixed-effect model was used to analyze the data $\left(I^{2}=0 \%, P=0.93\right)$. The results revealed obvious differences in ESR serum levels between TGP combined with MTX and those of MTX alone $(P<0.0001 ; \mathrm{MD}=-5.85$, $95 \% \mathrm{CI}=-8.67$ to -3.02$)$, as shown in Fig. $4 \mathrm{a}$.

Two trials reported the effects of the combination of TGP and MTX vs. MTX alone on CRP serum levels, and a random-effect model was used to analyze the data $\left(I^{2}=97 \%, P<0.00001\right)$. The results failed to find any significant differences in CRP serum levels between the two groups, however $(P=0.19 ; \mathrm{MD}=-8.62,95 \%$ $\mathrm{CI}=-21.44$ to 4.21$)$, as shown in Fig. $4 \mathrm{~b}$.

Four trials reported the effects of the combination of TGP and MTX vs. MTX alone on DMS, and a randomeffect model was used to analyze the data $\left(I^{2}=80 \%\right.$, $P=0.002)$. The results displayed no significant differences in the DMS between the two groups $(P=0.32$; $\mathrm{MD}=-9.16,95 \% \mathrm{CI}=-27.35$ to 9.02$)$, as shown in Fig. 4c.

Three trials reported the effects of the combination of TGP and MTX vs. MTX alone on TJC, and a fixed-effect model was used to analyze the data $\left(I^{2}=40 \%, P=0.19\right)$. The results failed to find any significant differences in TJC between TGP combined with MTX and that of MTX alone $(P=0.23 ; \mathrm{MD}=-0.61,95 \% \mathrm{CI}=-1.60$ to $0.38)$, as shown in Fig. 4d.

Three trials reported the effects of the combination of TGP and MTX vs. MTX alone on SJC, and a fixed-effect

Fig. 2 Risk of bias assessment

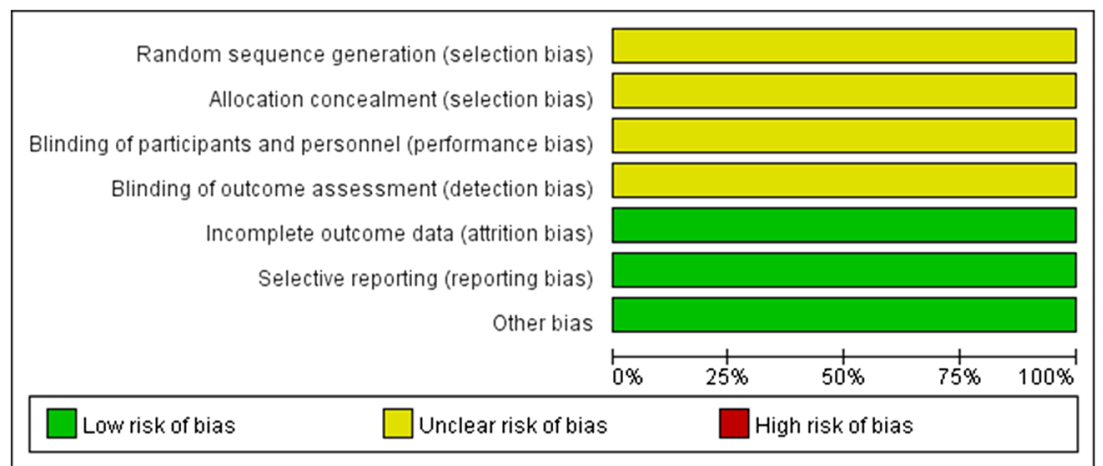




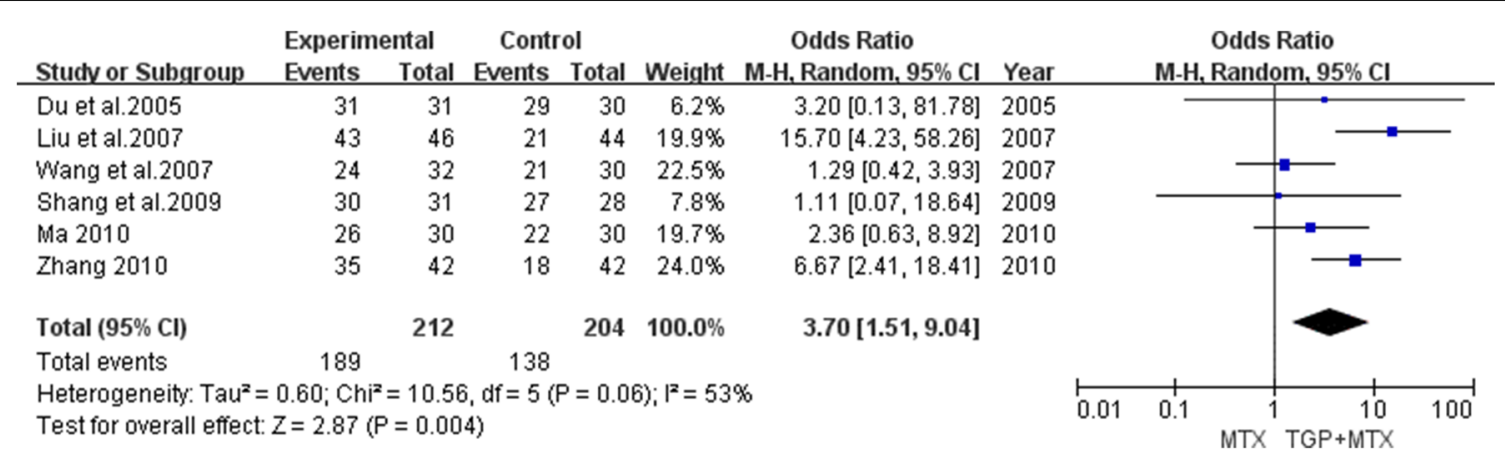

Fig. 3 Meta-analysis of the TEs of TGP combined with MTX vs. MTX alone. TE therapeutic effect, $T G P$ total glucosides of peony, $M T X$ methotrexate

model was used to analyze the data $\left(I^{2}=8 \%, P=0.34\right)$. The results displayed remarkable differences in SJC between TGP combined with MTX and MTX alone $(P<0.00001 ; \mathrm{MD}=-1.64,95 \% \mathrm{CI}=-2.27$ to -1.00$)$, as shown in Fig. 4e.

\section{AEs}

Seven studies reported AEs caused by TGP combined with MTX or MTX alone. AEs were not mentioned in one of the studies [28], but four out of seven trials [27, 29, 30, 34]

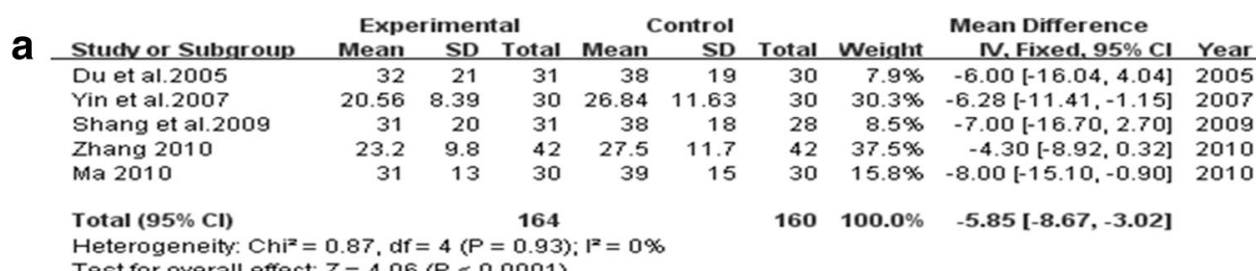

Test for overall effect: $Z=4.06(P \propto 0.0001)$
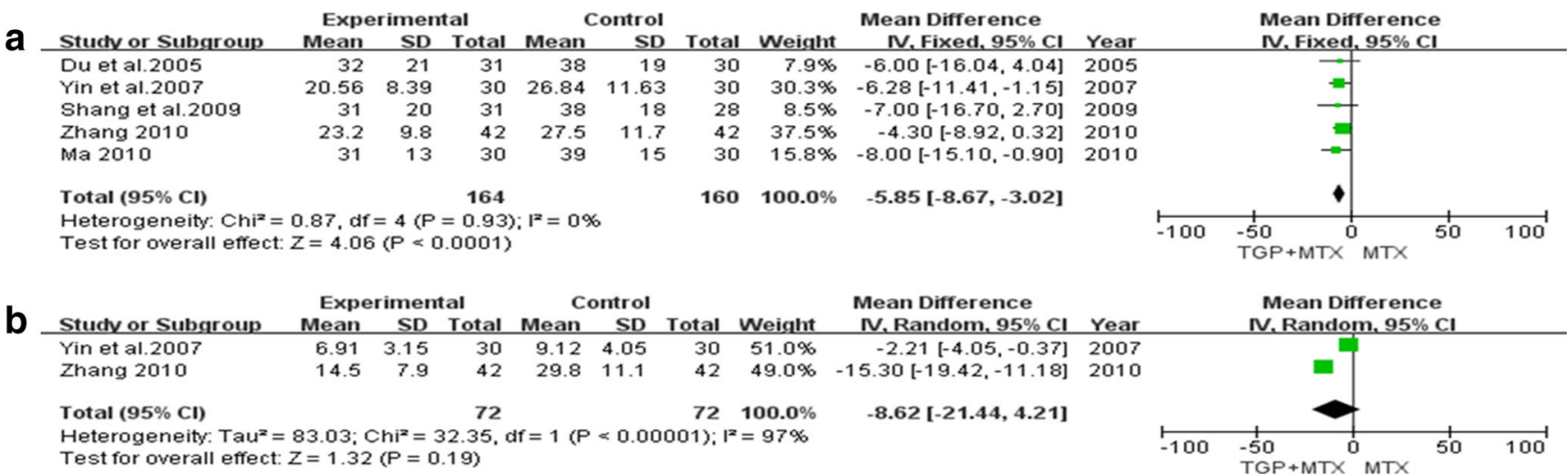

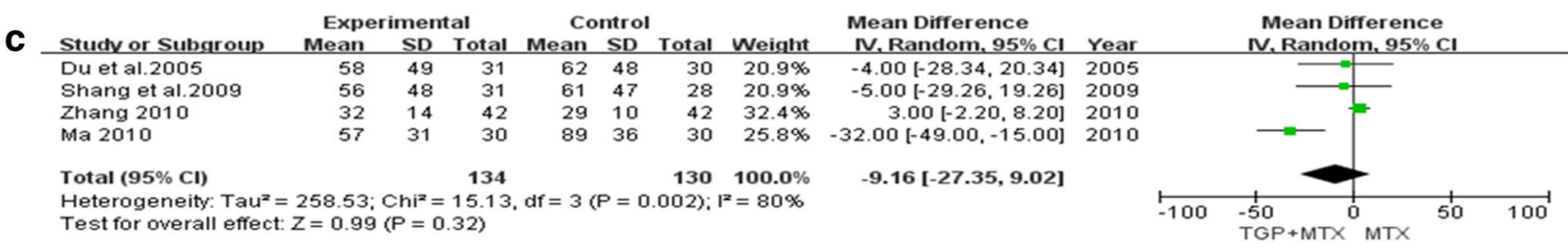

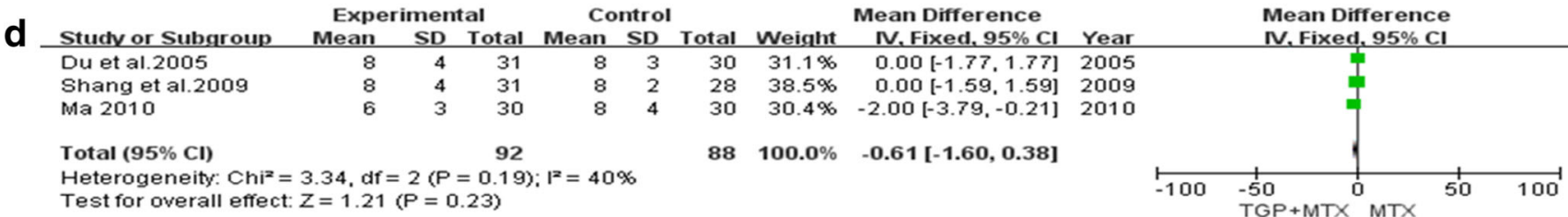

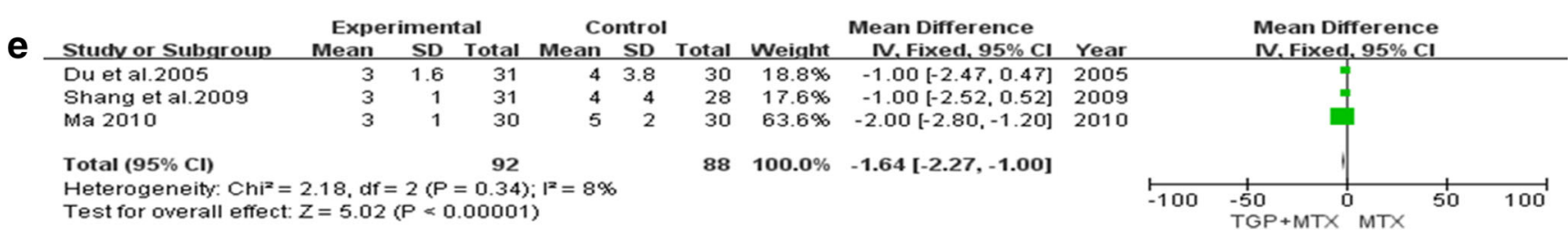

Fig. 4 Meta-analysis of the effects of the combination of TGP and MTX vs. MTX alone on serum levels of ESR, CRP, DMS, TJC, and SJC. a ESR. b CRP. c DMS. d TJC. e SJC. TGP total glucosides of peony, MTX methotrexate, ESR erythrocyte sedimentation rate, $C R P$ C-reactive protein, DMS duration of morning stiffness, TJC tender joint count, SJC swollen joint count 


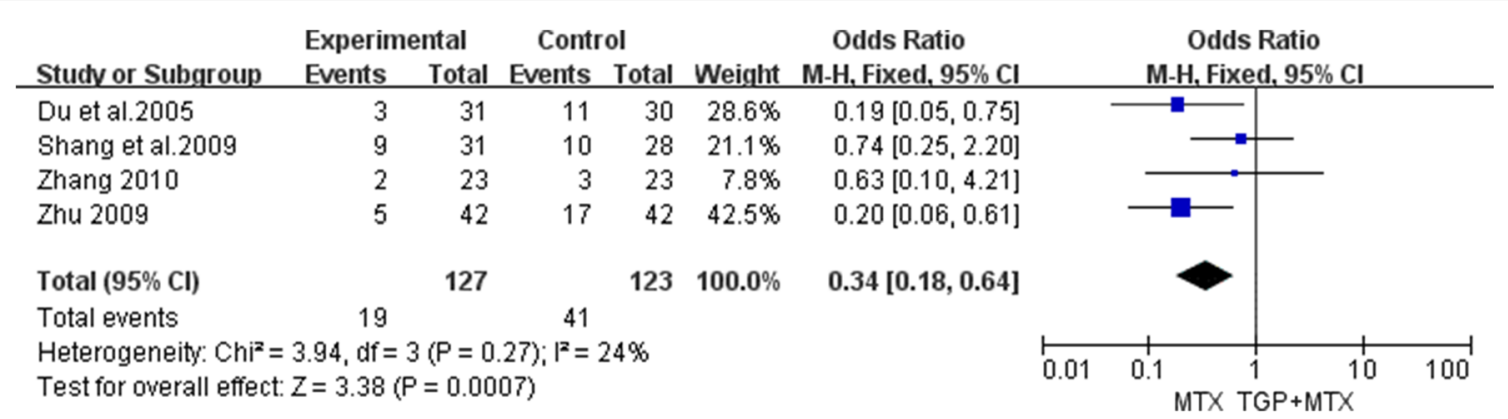

Fig. 5 Meta-analysis of AEs of TGP combined with MTX vs. MTX only for the treatment of RA. TGP total glucosides of peony, $M T X$ methotrexate, $A E$ adverse event

recorded $\mathrm{AE}$ cases in two groups. No heterogeneity was identified among the studies $\left(P=0.27\right.$ and $\left.I^{2}=24 \%\right)$ based on the fixed-effect model. As shown in Fig. 5, the results of our metaanalysis revealed a significantly higher rate of AEs induced by MTX alone than by TGP combined with MTX $(P=0.0007$; $\mathrm{OR}=0.34 ; 95 \%$ CI 0.18 to 0.64$)$. Four trials $[27,30,33,34]$ reported mild abnormal liver function, and five trials [27, 29, $30,33,34]$ recorded mild to moderate gastrointestinal events. These AEs mentioned above were relieved or disappeared after symptomatic treatment. No serious AEs were recorded.

\section{Publication bias}

Begg's publication bias plots showed that there were no significant publication biases when five or more studies were included (Fig. 6a, b). The results of the Egger test also showed that there was no significant publication bias (TEs, $P=0.825$; ESR, $P=0.306)$.

\section{Discussion}

This article provides a PRISMA-compliant meta-analysis and systematic review of the efficacy and safety of TGP combined with MTX in the treatment of RA. We previously investigated the efficacy and safety of the combination of TGP and leflunomide (LEF) on RA, and the results showed that TGP combined with LEF was more effective and safer than LEF alone for the treatment of RA [35]. In the present study, we included eight RCTs recording the TEs of TGP combined with MTX vs. those of MTX alone for the treatment of RA. A total of 265 patients in the treatment group (TGP combined with MTX) and 257 in the control group (MTX alone) were assessed. The pooled data suggests that TGP combined with MTX produces better therapeutic effects than MTX treatment alone. As shown in Fig. 4, the oral administration of TGP combined with MTX induced better effects on ESR and SJC than did MTX alone. Moreover, the pooled results indicate a significantly lower rate of AEs induced by TGP combined with MTX than was found in MTX alone.
Some related findings might help to explain the potential TEs of TGP combined with MTX in the treatment of RA. Previous pharmacological studies have concluded that TGP has crucial anti-inflammation, immunosuppression, and analgesic properties, as well as inhibiting bone destruction. TGP has been reported to possess anti-inflammatory effects by regulating pro-inflammatory mediators and suppressing the proliferation of fibroblast-like synoviocytes in collagen-induced
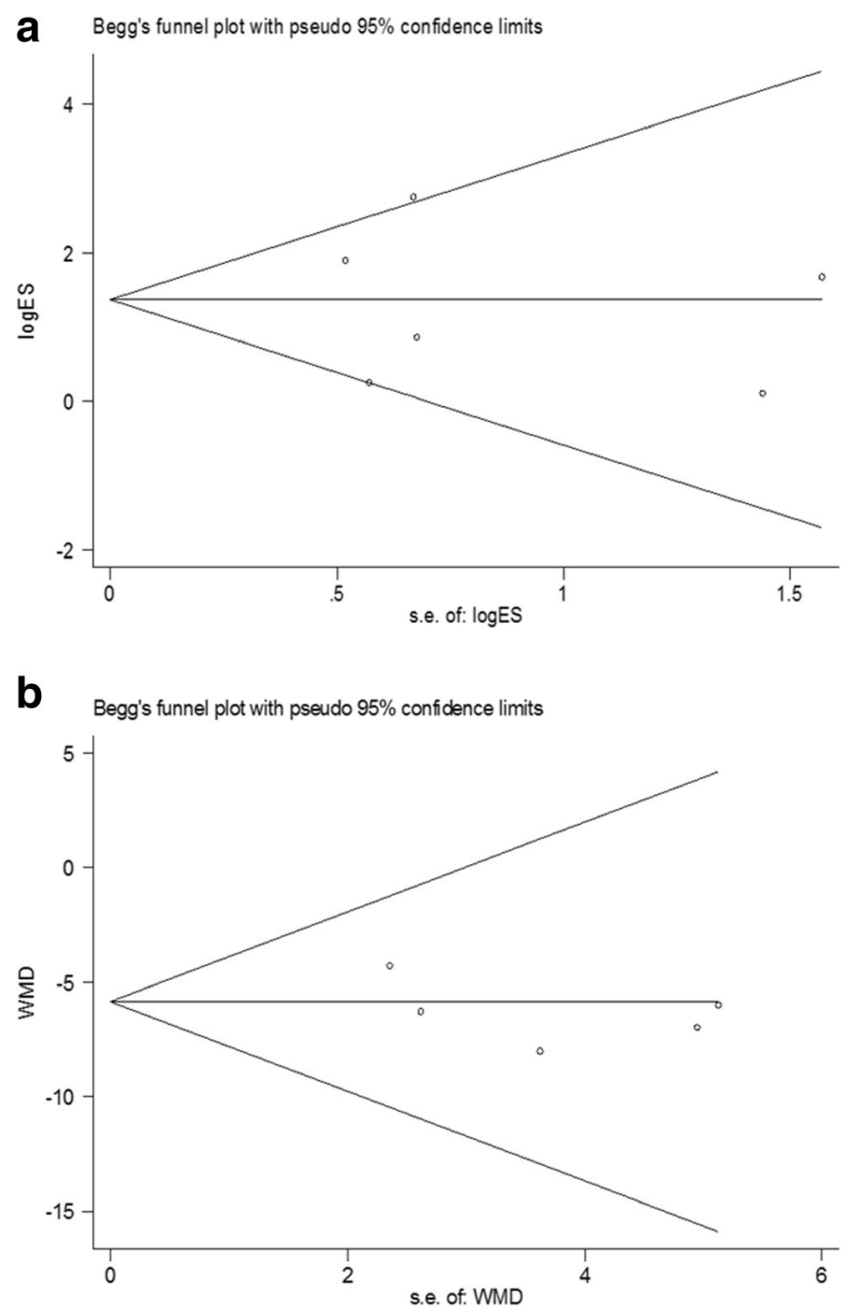

Fig. 6 The Begg funnel plots for publication bias in the included trials. a TE. b ESR. $T E$ therapeutic effect, $E S R$ erythrocyte sedimentation rate 
arthritis (CIA) or adjuvant arthritis (AA) model [13-16]. In addition, studies have shown that TGP inhibits both the maturation and function of dendritic cells (DCs) in vivo, which in turn results in reducing immune-mediated inflammation in vivo $[17,18]$. Moreover, paeoniflorin, the major active ingredient of TGP [12], has a prominent analgesic effect on visceral pain caused by colorectal distension in the rats with visceral hyperalgesia induced by neonatal maternal separation $[19,20]$. It is worth noting that TGP exerts a significant inhibiting effect on joint destruction in CIA [21] or antigeninduced arthritis (AIA) models [22].

Another, MTX is a folic acid antagonist and inhibits the synthesis of DNA, RNA, and proteins by binding to dihydrofolate reductase. Currently, MTX is the most widely used as a DMARD for the treatment of both early and established RA and has been named an "anchor agent" for combination therapy with other DMARDs and biological agents $[9,36]$. Several mechanisms have been proposed to explain the effects of MTX in RA, including the antagonism of folate-dependent processes, stimulation of adenosine signaling, generation of reactive oxygen species (ROS), downregulation of adhesion-molecule expression, regulation of cytokine profiles, and downregulation of eicosanoids and matrix metalloproteinases (MMPs) [36]. In the present study, we compared the TEs of the combination of TGP and MTX to those of MTX alone for the treatment of RA, and the results indicated that the combination group exhibited efficacy than the monotherapy group, suggesting that TGP could be used as a type of "herbal DMARD" for complementary RA therapeutics.

Nonetheless, our meta-analysis has several limitations which should be taken into consideration when interpreting our results. First, all of the included studies were conducted in Chinese populations, which presented a high risk of selection bias. Second, the quality scoring system of the included trials was not well established. The randomization procedures were not described in all eight studies. In addition, the general lack of information on allocation concealment, blinding, dropouts, and intention to treat may conceal potential selection or detection biases. Third, publication bias is possible because all of the studies were published in Chinese, although we did conduct comprehensive searches in both English and Chinese publication databases for relevant articles. Fourth, the outcome measures were inadequate, and only one study described the clinical outcomes defined by the American College of Rheumatology (ACR) criteria [30]. Besides that, differences in the quality of trials, intervention methods, doses, and treatment duration were responsible for the heterogeneity. Based on the above, we suggest that future researchers focus on the following criteria. RCTs of the efficacy and safety of TGP combined MTX in treating RA need larger sample sizes, multiple centers, and longer follow-up times. And RCTs should describe their randomization, allocation concealment, blinding, and other information. Moreover, researchers should formulate strict inclusion and exclusion criteria and standardize both outcome evaluation indicators and safety analysis. Consequently, the conclusions of this study should be carefully interpreted.

\section{Conclusions}

In summary, the results of the present study suggest that TGP combined with MTX is more effective than MTX alone for the treatment of RA. Therefore, TGP can be used as a complementary DMARD. In addition, the AEs should be further evaluated. Given that the quality of included trials was moderate to low, well-designed, multi-center, and large-scale RCTs are necessary to further confirm our results.

Acknowledgments This project was supported by grants from the Guangdong Provincial Natural Science Foundation (2015A030310067), Guangdong Science and Technology Program (2016A020226001), and Shenzhen Technology Innovation Foundation (JCYJ20150401171352927).

Authors' contributions Juan Li and Zhi-gang Mei conceived the study. Zhi-tao Feng and Juan Xu performed literature searches and study selection. Guo-chao He and San-jin Cai performed data extraction and quality assessment. Zhi-tao Feng and Juan Xu performed the statistical analyses and prepared the manuscript. Zhi-tao Feng, Juan Li, and Zhigang Mei critically revised the manuscript.

\section{Compliance with ethical standards}

\section{Disclosures None.}

Open Access This article is distributed under the terms of the Creative Commons Attribution 4.0 International License (http:// creativecommons.org/licenses/by/4.0/), which permits unrestricted use, distribution, and reproduction in any medium, provided you give appropriate credit to the original author(s) and the source, provide a link to the Creative Commons license, and indicate if changes were made.

\section{References}

1. Smolen JS, Aletaha D (2015) Rheumatoid arthritis therapy reappraisal: strategies, opportunities and challenges. Nat Rev Rheumatol 11(5):276-289. doi:10.1038/nrrheum.2015.8

2. McInnes IB, Schett G (2011) The pathogenesis of rheumatoid arthritis. N Engl J Med 365(23):2205-2219. doi:10.1056/ NEJMra1004965

3. Kourilovitch M, Galarza-Maldonado C, Ortiz-Prado E (2014) Diagnosis and classification of rheumatoid arthritis. J Autoimmun 48-49:26-30. doi:10.1016/j.jaut.2014.01.027

4. Hunt L, Emery P (2014) Defining populations at risk of rheumatoid arthritis: the first steps to prevention. Nat Rev Rheumatol 10(9): 521-530. doi:10.1038/nrrheum.2014.82

5. Koenders MI, van den Berg WB (2015) Novel therapeutic targets in rheumatoid arthritis. Trends Pharmacol Sci 36(4):189-195. doi:10. 1016/j.tips.2015.02.001 
6. Calabro A, Caterino AL, Elefante E, Valentini V, Vitale A, Talarico R, Cantarini L, Frediani B (2016) One year in review 2016: novelties in the treatment of rheumatoid arthritis. Clin Exp Rheumatol 34(3):357-372

7. Siebert S, Tsoukas A, Robertson J, McInnes I (2015) Cytokines as therapeutic targets in rheumatoid arthritis and other inflammatory diseases. Pharmacol Rev 67(2):280-309. doi:10.1124/pr.114. 009639

8. Lee YH, Bae SC, Song GG (2011) The efficacy and safety of rituximab for the treatment of active rheumatoid arthritis: a systematic review and meta-analysis of randomized controlled trials. Rheumatol Int 31(11):1493-1499. doi:10.1007/s00296-010-1526-y

9. Cipriani P, Ruscitti P, Carubbi F, Liakouli V, Giacomelli R (2014) Methotrexate: an old new drug in autoimmune disease. Expert Rev Clin Immunol 10(11):1519-1530. doi:10.1586/1744666X.2014. 962996

10. Zhang P, Li J, Han Y, Yu XW, Qin L (2010) Traditional Chinese medicine in the treatment of rheumatoid arthritis: a general review. Rheumatol Int 30(6):713-718. doi:10.1007/s00296-010-1370-0

11. Moudgil KD, Berman BM (2014) Traditional Chinese medicine: potential for clinical treatment of rheumatoid arthritis. Expert Rev Clin Immunol 10(7):819-822. doi:10.1586/1744666X.2014. 917963

12. Zhang W, Dai SM (2012) Mechanisms involved in the therapeutic effects of Paeonia lactiflora Pallas in rheumatoid arthritis. Int Immunopharmacol 14(1):27-31. doi:10.1016/j.intimp.2012.06.001

13. Xu HM, Wei W, Jia XY, Chang Y, Zhang L (2007) Effects and mechanisms of total glucosides of paeony on adjuvant arthritis in rats. J Ethnopharmacol 109(3):442-448. doi:10.1016/j.jep.2006. 08.019

14. Chang Y, Wei W, Zhang L, Xu HM (2009) Effects and mechanisms of total glucosides of paeony on synoviocytes activities in rat collagen-induced arthritis. J Ethnopharmacol 121(1):43-48. doi: 10.1016/j.jep.2008.09.028

15. Jia XY, Chang Y, Sun XJ, Wu HX, Wang C, Xu HM, Zhang L, Zhang LL, Zheng YQ, Song LH, Wei W (2014) Total glucosides of paeony inhibit the proliferation of fibroblast-like synoviocytes through the regulation of $\mathrm{G}$ proteins in rats with collagen-induced arthritis. Int Immunopharmacol 18(1):1-6. doi:10.1016/j.intimp. 2013.09.007

16. Zheng YQ, Wei W (2005) Total glucosides of paeony suppresses adjuvant arthritis in rats and intervenes cytokine-signaling between different types of synoviocytes. Int Immunopharmacol 5(10):15601573. doi:10.1016/j.intimp.2005.03.010

17. Zhou Z, Lin J, Huo R, Huang W, Zhang J, Wang L, Sun Y, Shen B, Li N (2012) Total glucosides of paeony attenuated functional maturation of dendritic cells via blocking TLR4/5 signaling in vivo. Int Immunopharmacol 14(3):275-282. doi:10.1016/j.intimp.2012.07. 012

18. Lin J, Xiao L, Ouyang G, Shen Y, Huo R, Zhou Z, Sun Y, Zhu X, Zhang J, Shen B, Li N (2012) Total glucosides of paeony inhibits Th1/Th17 cells via decreasing dendritic cells activation in rheumatoid arthritis. Cell Immunol 280(2):156-163. doi:10.1016/j. cellimm.2012.12.005

19. Zhang XJ, Chen HL, Li Z, Zhang HQ, Xu HX, Sung JJ, Bian ZX (2009) Analgesic effect of paeoniflorin in rats with neonatal maternal separation-induced visceral hyperalgesia is mediated through adenosine $\mathrm{A}(1)$ receptor by inhibiting the extracellular signalregulated protein kinase (ERK) pathway. Pharmacol Biochem Behav 94(1):88-97. doi:10.1016/j.pbb.2009.07.013
20. Zhang XJ, Li Z, Leung WM, Liu L, Xu HX, Bian ZX (2008) The analgesic effect of paeoniflorin on neonatal maternal separationinduced visceral hyperalgesia in rats. J Pain : Off J Am Pain Soc 9(6):497-505. doi:10.1016/j.jpain.2007.12.009

21. Zhu L, Wei W, Zheng YQ, Jia XY (2005) Effects and mechanisms of total glucosides of paeony on joint damage in rat collageninduced arthritis. Inflamm Res : Off J Eur Histamine Res Soc 54(5):211-220. doi:10.1007/s00011-005-1345-X

22. Wei CC, You FT, Mei LY, Jian S, Qiang CY (2013) Total glucosides of paeony prevents juxta-articular bone loss in experimental arthritis. BMC Complement Altern Med 13:186. doi:10.1186/14726882-13-186

23. Arnett FC, Edworthy SM, Bloch DA, McShane DJ, Fries JF, Cooper NS, Healey LA, Kaplan SR, Liang MH, Luthra HS et al (1988) The American Rheumatism Association 1987 revised criteria for the classification of rheumatoid arthritis. Arthritis Rheum 31(3):315-324

24. Jadad AR, Moore RA, Carroll D, Jenkinson C, Reynolds DJ, Gavaghan DJ, McQuay HJ (1996) Assessing the quality of reports of randomized clinical trials: is blinding necessary? Control Clin Trials 17(1):1-12

25. DerSimonian R, Laird N (1986) Meta-analysis in clinical trials. Control Clin Trials 7(3):177-188

26. Higgins JP, Thompson SG, Deeks JJ, Altman DG (2003) Measuring inconsistency in meta-analyses. BMJ 327(7414):557560. doi:10.1136/bmj.327.7414.557

27. Zhang Z (2010) Study on clinical efficacy of methotrexate combined with total glucosides of paeony in the treatment of rheumatoid arthritis. Chin J Urban Rural Enterp Hyg 3:24-25

28. Ma J (2010) Study on clinical efficacy of methotrexate combined with total glucosides of paeony in treating rheumatoid arthritis. Jilin Med J 31(28):4934-4935

29. Shang GL, Liu JH (2009) Comparative study on clinical efficacy of methotrexate combined with total glucosides of paeony in the treatment of rheumatoid arthritis. Chin J Clin Ration Drug Use 2(4):1-2

30. Zhu J (2009) Clinical analysis of methotrexate combined with total glucosides of paeony in treating rheumatoid arthritis. Shanxi J Tradit Chin Med 25(sup):48-49

31. Liu ZY, Liu XJ, Wang ZH (2007) Study on clinical efficacy of pafulin combined with methotrexate in the treatment of rheumatoid arthritis. Cent Plains Med J 34(3):83-84

32. Wang DF, Pei RX (2007) Total glucosides of paeony treated 32 patients with rheumatoid arthritis. Shanxi J Tradit Chin Med 28(4):421-422

33. Yin G, Xie QB (2007) Clinical analysis of the efficacy of total glucosides of peony capsules on rheumatoid arthritis. Mod Prev Med 34(19):3791-3794

34. Du JH, Dong BD (2005) Comparative study on clinical efficacy of using methotrexate singly or combined with total glucosides of paeony in treating rheumatoid arthritis. Chin J Integrated Tradit West Med 25(6):540-542

35. Feng Z, Xu J, He G, Cao M, Duan L, Chen L, Wu Z (2016) The efficacy and safety of the combination of total glucosides of peony and leflunomide for the treatment of rheumatoid arthritis: a systemic review and meta-analysis. Evid Based Complement Alternat Med : eCAM 2016:9852793. doi:10.1155/2016/9852793

36. Brown PM, Pratt AG, Isaacs JD (2016) Mechanism of action of methotrexate in rheumatoid arthritis, and the search for biomarkers. Nat Rev Rheumatol 12(12):731-742. doi:10.1038/nrrheum.2016.175 